\title{
ON THE DYSENTERY TOXIN.
}

\author{
By Dr. H. LüDke, Barmen, Germany.
}

Is connection with the protection of the organism against microorganisms and their toxins, numerous experiments have now been made. These experiments have aimed, on the one hand, at perfecting the efficacious components of the protoplasm of the bacilli, and, on the other, at producing extremely virulent extracellular toxins. An attempt has been made, in order to secure active immunity, to cultivate most virulent cultures of various bacilli. As an example, I may cite the effects of active immunisation against the typhoid bacillus made during the South African War by Wright, who, although he lays great stress upon the value of virulent cultures, does not omit to mention the disagreeable secondary effects-such as headache, nausea, violent pain in the joints, rise of temperature, infiltrations at the seat of injection-after the injection of small doses of living cultures. These troublesome secondary effects remained almost unaltered when, following the recommendation of Wassermann, Kitasato, and Brieger, macerated cultures were used instead of living organisms, though one single injection of this macerated culture was sufficient for the production of bacteriolytic serum; and the proceeding offered, in its practical application, an important safeguard for the physician.

Not long ago it was recognised that virulence is not the sole factor to be considered in the production of immunity; it was soon found that the faculty of producing immunity has its existence not merely in the micro-organisms, but that, as a matter of fact, specific cells are the producers of specific amboceptors. The nature of this specific process of cell secretion is still unknown to us. However, after proving the existence of products of the cell action in the serum, a new way was opened up for arriving at new conceptions and grasping laws which afford a better explanation than has hitherto been obtainable of the bio-chemical process of immunity, the principal characteristic of which -it must once more be pointed out-consists in the specific action of particular cells, as in typhoid fever for instance, in the action of the blood-producing organs. We therefore had a new law when, as regards typhoid fever, Wassermann proved that virulence is not the determining factor in the production of immunity, and that a way more likely to lead to successful results was indicated by the quantitative 
analysis of the combining power of specific bacilli for the amboceptors. In this matter Wassermann has been guided by the views of Ehrlich, who assumes that the substances secreted as the result of reaction are the independent receptors possessed of a particular sphere of action in the specific cells. The natural outcome was an attempt to obtain a quantitative measure of this specific production of amboceptors in the blood serum, by combining certain races of a greater or less degree of virulence. In this respect, however, it still seems doubtful whether the observations made by Strong, working with Wassermann, can be generally accepted. Strong maintains that he could make out no difference in the faculty of combination in the sensible cells of any species of animals, the same quantity of amboceptors, proportionally, being absorbed in the serum of different animals. In my opinion, however, the adequate stimulus may meet with differentiated and individually varied proportions in the secreting cells, even for the same species, and the more so for different species, so that I cannot consider the results obtained by Strong as generally available.

We must bear in mind the maxim that only the "specific" cells of the organism are capable of the function of secretion consequent upon an adequate stimulus, and this must also be borne in mind in the case of the antitoxins. With regard to the production of the latter, we have likewise to take into consideration the specific property of the protoplasm, which may vary according to the race of animals, to the general condition of nutrition, to the sensibility of the secreting cells, and to the toxic activity of the bacilli. The property of influencing the protoplasm of the specific cells must be variable in the process of immunisation, as sufficient examples have demonstrated to us.

Originally, special attention was paid to the production of a rather strong and abundant toxin, and in two infectious diseases, diphtheria and tetanus, the production of a specially efficacious toxin was obtained. In the case of bacillary dysentery, of which these preliminary communications treat, very few experiments for the purpose of obtaining toxin have, up to the present, been made.

Commencing with the researches of Kruse, the German discoverer of the Bacillus dysenterice, we find in one of his essays ${ }^{1}$ the assertion that it is impossible to obtain very strong toxins from the $B$. dysenterice.

Conradi ${ }^{2}$ agrees with him in this, because the latter's injections of filtered eight or fourteen or thirty days old cultures, whether made subcutaneously, intraperitoneally, or intravenously, and given in large doses, did not result in the death of the guinea-pigs or rabbits that he used for this purpose. Vaillard and Dopter ${ }^{3}$ obtained similar results. Consequently, nothing further was to be expected from the production of toxin by the methods hitherto used. I have verified the correctness of the preceding observations by the injection into rabbits and white mice of cultures several days old.

Lately a new method of producing the toxin of this bacillus has been

${ }^{1}$ Deutsche med. Wchnschr., Leipzig, 1903, S. 6.

2 lbid., S. 27.

${ }^{3}$ Ann. de l'Inst. Pastcur, Paris, 1903, tome xvii. r. 463. 
resorted to. Conradi was the first to indicate the following procedure:Aseptic autolysis. Twenty-hour agar cultures of the dysentery bacillus were taken, the surface growth was mixed with physiological salt solution, and maintained for one or two days at $37^{\circ} \mathrm{C}$. This solution absorbed the active substances and, after filtration through porcelain filters and concentration in the vacuum apparatus, yielded a toxin soluble in water which, when injected in doses of 0.1 c.c., killed a rabbit in twenty-four hours. Shiga and Neisser ${ }^{1}$ followed the same principle, but with the practically improved method of using filtered extracts (obtained by heating), 0.5 to 0.2 c.c. of which was fatal to a rabbit in two days.

Rosenthal, ${ }^{2}$ Moscow, prefers the old method of natural production of toxins, but uses Martin's weak alkaline bouillon, and allows the growth to go on for about three weeks; he then, at $37^{\circ} \mathrm{C}$., produced a toxin; $0 \cdot 2$ to $0 \cdot 1$ c.c. filtered culture of this injected subcutaneously is sufficient to kill a rabbit of $4 \frac{1}{2} \mathrm{lbs}$. in twenty-four to forty-eight hours. The clinical symptoms correspond to those following an infection with bacilli, namely, paresis, first of the hindlegs and then of the fore-legs, frequent diarrhoea, great and rapid fall of temperature, rapid decrease of weight and collapse. Among the pathological symptoms I have noticed the following as being of special importance :Flatulence of the great gut, here and there hæmorrhagic infiltrations with slight necrosis of the gut, great distension of the bladder. Rosenthal also mentions hyperæmia of the small intestine, and the presence of mucous fluid sometimes mixed with blood. The result obtained by Rosenthal is important as regards treatment, as he obtained a protection against the infection through bacilli by the serum of the animals treated with the toxin prepared by him.

Considering the above-mentioned observations on dysentery toxin, we find two views hitherto held concerning the production of soluble toxins from bacteria, which broadly belong to the prototype of the $B$. typhi abdominalis. The researches on the $B$. dysenterice seem to indicate that we cannot refer the active substance to endotoxins as opposed to the soluble products of secretion. There appears to be a possibility of obtaining the dysentery toxin both as a simple product of secretion and as an endo-toxin, though, according to the generally accepted view, we should mention in this connection only the endotoxins.

Rodet, Lagriffoul, and Wahby appear to have succeeded, as recently stated, ${ }^{3}$ in obtaining a toxin in culture-filtrates from the B. typhi abdominalis by means of very young cultures in bouillon of high alkalinity. They have, however, to use comparatively large doses. Generally speaking, it seems to be no longer doubtful that in bouillon a toxin is present as the product of secretion even in the case of the $B$. typhi abdominalis, if young cultures are used and a sufficient supply of air is allowed to gain access to them. The bacilli collected on the filter are, according to the above authors, not more virulent than the filtrate, so that the idea that the $B$. typhi abdominalis was virulent solely because of its intracellular endotoxins adhering to the protoplasm must naturally lose ground, though there still remains the difficulty of getting that considerable part of toxin incorporated in the bacillus thrown out into the bouillon. The possibility of obtaining a complete gain of this toxin is in principle, however, still open to us.

A valuable addition to the method of obtaining virulent substances from the bodies of bacteria has been given in various publications by Macfadyen and Rowland. ${ }^{4}$ By means of a complicated and ingenious apparatus these authors, using low temperatures, have succeeded in obtaining virulent extracts from the protoplasm of specific bacteria. The proceeding, briefly stated, was as follows :

\footnotetext{
1 Deutsche med. Wchnschr., Leipzig, 1903, S. 61.

2 Ibid., 1904, S. 235.

${ }^{3}$ Centralbl. f. Bakteriol. u. Parasitenk., Jena, 1904, Bd. xxxvi., Orig., S. 593.

4 Ibid., Jena, 1903, Bd. xxxiv., Orig., SS. 618, 765.
} 
-The typhoid bacilli, frozen by being dipped into a vessel of liquid air, were crushed in a triturating apparatus worked by electricity. The triturated cells were then transferred to physiological salt solution, and freed from any adhering matter by careful centrifugalisation. With this cell juice Macfadyen and Rowland obtained a toxic effect on the animal organism far more active than that of the most virulent culture. Experiments were also made with Streptococcus pyogenes, Staphylococcus pyogenes aureus, B. enteritidis (Gärtner), $B$. tuberculosis, and $B$. diphtherice, all of which yielded the same toxic cell extracts.

My experiments to obtain such toxic products from the bodies of bacteria have been carried out on the dysentery bacillus of Kruse. As I had not so complicated an apparatus as that used by Macfadyen and Rowland at my disposal, I tried by means of manual trituration to obtain as complete disintegration as possible of bacteria treated with liquid air. Similar experiments have recently been made by Basseuge and Mayer ${ }^{1}$ after I had commenced mine. They wished to obtain a toxin from the typhoid bacillus, but their experiments were attended with no marked success, the virulence of the cell juices thus obtained not corresponding to that obtained by Macfadyen, whilst the antibacterial and antitoxic property of the serum of rabbits treated with this toxin was in no degree striking.

The following is a somewhat detailed account of the method I employed :-

The experiments which I first carried out in the Farbenfabriken, late Fr. Bayer \& Co., Elberfeld, with the kind assistance of Dr. Wesenberg, consisted of a trituration of bacteria in a compression machine placed in liquid air; these were unsuccessful. Several new experiments were then undertaken in order to obtain dysentery endotoxin from the dysentery bacilli by manual trituration in a mortar. These experiments had to be undertaken with the utmost caution, as a part of the bacteria used were living. ${ }^{2}$

Agar cultures, in large and small Petri dishes and in Kolle's bottles (on a surface of 100 to $250 \mathrm{cms}$.), were inoculated from a stock of dysentery bacilli kindly sent to me by Professor Kruse, Bonn. They were left in the incubator for twenty-four to thirty-six hours at $37^{\circ} \mathrm{C}$. ( $\frac{1}{20}$ agar culture of this stock killed a medium-sized rabbit in forty-eight hours). Then the growth was washed off with a physiological salt solution, and the emulsion being dried in the vacuum-drying apparatus at room temperature, there resulted a compound of a greyish-yellow colour, glittering and schistoid. The quantity of this product, dried as indicated, after being previously separated from any of the agar-culture medium by means of long and careful centrifugalisation, was always exceedingly small, seldom more than $0.1 \mathrm{grm}$. The dried bacteria were often poured over liquid air and then triturated with the pestle, the strongest manual pressure being used. The proceeding was continued until few intact bacilli were visible in a microscopic preparation. To the pasty mass thus obtained a sterile solution of common salt of about 20 to

${ }^{1}$ Centralbl. f. Bakteriol. u. Parasitenk., Jena, 1904, Bd. xxxvi.

2 It was unavoidable that the writer had to try to infect himself, such infection resulting in a series of disagreeable symptoms associated with a slight dysentery, which lasted for about ten days. 
40 c.c. was added, and to free the compound from any germs that might have got into it during the various processes, it was filtered through a Pukall's filter. A sterile liquid as clear as water, which seomed fit for injection, was thus obtained. $U_{p}$ to the present $I$ have experinented upon a good number of animals, chiefly rabbits, and the injection with the sterile cell juice was, as a rule, made into the veins. On the whole, $I$ achieved pretty constant. results; for instance, injections of 0.5 to 0.2 c.c. of this toxic liquid sufficed to kill a rabbit of $3 \frac{1}{12}$ lbs. weight in eighteen to twenty-four hours; an injection of 0.1 c.c. caused the death of the test aninal within forty-eight hours in some cases, and in others it produced a series of exceedingly characteristic symptoms analogous to those produced by injections of living or of macerated dysentery bacilli. I obtained the best result by means of an intravenous injection of 0.05 c.c. cell juice; this caused the death of the animal in thirty-six hours. Guinea-pigs which received an intraperitoneal injection of 0.5 to 0.1 c.c. also llied within one day, or two at the latest. Rats of about 6 to $8 \mathrm{oz}$. in weight died within a still shorter period. According to my observations, doses varying from 0.1 to 0.05 c.c. might be insufficient to kill a well developed rabbit, though it usually remained in a very bad state of health.

The clinical symptoms before death, and even after sublethal doses, were as follows :-

Constant decrease in weight of the animals injected, amounting sometimes to $T$ oz. for animals of about $3 \mathrm{lb}$. in weight; a rapid fall of temperature took place, of $2^{\circ}$ to $3^{\circ} \mathrm{C}$., in most cases ; diarrhoa was only noted in the case of a small number of animals; paresis of the legs, beginning in the hind-legs, showed itself after about eighteen to twenty-four hours.

We may see from the above that the clinical symptoms correspond exactly to those observed when living or macerated bacteria are injected.

On post-mortem examination the following conditions were found :-There was little alteration in the serous membrane of the peritoneal cavity, rarely some hyperæmic areas; there was no turbid or bloody exudation in this cavity, and the fluid found was sterile. The liver, spleen, kidney, heart, and other organs showed no lesion on macroscopical examination; a more conspicuous feature was the great distension of the small intestine as compared with the great gut. Hyperamia of the mucous membrane of the intestine and hæmorrhages into the mucosa of the great gut were observerl in very few cases. I have never found any abscess at Bauhin's valve or in the great gut. On subcutaneous injections only a toxic celema was observed at the seat of injection. The potency of these sterile cell juices seemed to be strictly limited as to time, for after this liquid had been preserved in an ice-box for eight days a far greater dose was required to kill an animal.

A further and far more inportant fact in connection with the practical use of these toxic cell juices for the production of inmunity seems to me to consist in the dose required, and here arises a contrast between my results and those obtained by Macfadyen and Rowland. After injection of a sublethal dose which caused distinct symptoms of disease, a similar dose killed the test animal in a shorter time, and I could find no anti-bacterial or antitoxic properties in the blood serum of the animals so treated. Up to now I have been unsuccessful in obtaining any agglutinating property of the serum after a single sublethal dose, but it was interesting to note the strong 
reaction of leucocytes, a distinct hyperleucocytosis following a subcutaneous injection; the particulars of this will be given later.

On thoroughly considering the results obtained, I am compelled to come to conclusions that differ in certain respects from those arrived at by Macfadyen and Rowland. There can be no doubt that the improved method of producing toxin indicated by these authors has great advantages, in so far that it is now possible to obtain soluble extracts in an almost pure state. There is a further advantage in the fact that such soluble cell juices bring about a fatal result far more rapidly than do the most virulent infections of bacteria, consequently their toxicity is fully demonstrated. The old methods of obtaining cell juices in sufficient concentration and efficaciousness are therefore improved upon. The action on the organs producing amboceptors is here more rapid and more intensive than where the whole cells are used, so that through the specific stimulus in giving the cell protoplasm its specific direction an excessive action of the cells is promoted, that is to say, a successful secretion of receptors can take place.

Comparatively speaking, there is a certain accordance between the experiments of Macfadyen and Rowland and those of Conradi, Neisser, and Shiga, as both sets of workers have followed physiological lines, namely, the disintegration of the whole cell within the organism, and setting free the toxin-bearing, soluble substratum that is characteristic of the cell outside the animal body.

As yet, however, in my opinion, those endotoxins cannot be used practically in connection with the production of antitoxins. As the result of several investigations, I have convinced myself that injections of such active cell juices for the purpose of immunisation determine an increase in the sensibility of the organism, with the result that no antitoxin, or rather no antiendotoxins, can be formed, for it is of endotoxins only that we are entitled to speak in connection with the above cases.

Not long ago A. Wolff ${ }^{1}$ very justly called attention to the fact that, by means of endotoxins obtained in different manners, it is impossible for the organism to produce a blood serum capable of neutralising the endotoxins set free by the injection of bacteria. The principal effect of the injection of juices containing endotoxins consists solely in the setting free of amboceptors, which in turn, in case of a new infection of bacteria, set free new endotoxins from the bacteria.

The cell juices obtained by the method of Macfadyen and Rowland seem to be specially associated with the acquisition of a strong and active immunity, owing to the intensity and rapidity of their action as well as to their dose being easily graduated.

I am indebted to Dr. Markwald, director of the Bacteriological Institute, Barmen, for so kindly placing at my disposal the appliances and apparatus of the Institute.

1 Berl. klin. Wchnschr., 1904, SS. 1105, 1131, 1156. 